\title{
Systematizing the Service-oriented Requirements Engineering Through i* Framework and Web Services
}

\author{
Keith de Souza, Marcelo Fantinato, Marcelo Medeiros Eler \\ School of Arts, Science and Humanities University of São Paulo - USP \\ São Paulo - SP, Brazil \\ \{keith, m.fantinato, marceloeler\}@usp.br
}

\begin{abstract}
Owing to the dynamicity of business environments in which organizations must quickly adapt to changes, the information systems have recently had to adapt to new situations so that they can keep adding value efficiently and effectively. In the light of this scenario, a new discipline called Service-oriented Systems Engineering has emerged in the academic scene and this has highlighted the disciplined, systematic and quantified development of Service-oriented Computing systems. This discipline is divided into other subdisciplines; one of these sub-disciplines is Service-oriented Requirements Engineering (SORE) and it is concerned with defining processes and methodologies to address the question of services requirements from two different perspectives: service consumer and service provider. In the SORE context, this paper proposes the WS\&i*-RGPS approach that explores some alternatives to the descriptions proposed by the Role, Goal, Process and Service (RGPS) metamodels - an approach in SORE. This involves a new definition that seeks to incorporate the benefits of other methodologies - established in the literature - with RGPS. Accordingly, this new approach outlines the use of the $i^{*}$ Framework to describe the Role and Goal layers and shows the use of WS-BPEL/WSDL to describe the process and service layers. The use of the $i *$ Framework sets out the goals for managing different aspects of the systems specification. Moreover, this paper presents a comparison among WS\&i*RGPS and other SORE approaches based in three parameters presented in SORE literature.
\end{abstract}

Keywords-service-oriented requirement engineering; SORE; RGPS; $i$ * Framework; WS-BPEL.

\section{INTRODUCTION}

In recent years, organizations have had to face a highly dynamic business environment where the basic needs are changing more frequently than ever due to the high degree of competition that exists between organizations [1]. Apart from organizational changes, the information systems must be quickly adapted to the different circumstances within the organization so that it can retain its benefit. In the light of this scenario, the novel computing paradigm, called Serviceoriented Computing (SOC), uses services as a basic means of supporting the rapidly and low cost development of distributed and easy to compose applications even when they are running in heterogeneous environments [2].

Service Oriented Systems Engineering (SOSE) is a new discipline that has emerged to develop, operate and maintain systems based on the SOC paradigm. SOSE is divided into other sub-disciplines. One of them is Service-oriented
Requirements Engineering (SORE), that defines processes and methodologies to capture, elicit and specify the services requirements from two different standpoints: service consumer and service provider [3].

In the mid-2000s, Papazoglou et al. [2] and Kontogiannis et al. [4] carried out researches in which SOSE was highlighted as a challenge that needs to be solved and a yet unexplored field. However, $\mathrm{Gu}$ and Lago [5] showed that there had been an increase of published works between 2006 and 2009 that addressed the issues and challenges raised by SORE. Therefore, SORE emerged as an important discipline in the context of SOC because of the key role played by requirements engineering in the systems development life cycle [6]. The number of published works in SORE increased in the middle of 2007 with regard to these two main points: $(i)$ the extension of techniques in traditional requirements engineering (for instance, new proposals that employed current techniques as the $i *$ Framework [7], [8], [9] emerged); and (ii) novel techniques designed especially for SOC (for instance, the definition of metamodels Role, Goal, Process and Service - RGPS [6], [10]).

The RGPS metamodels can be defined as a hierarchical and cooperative approach based on four layers: Role, Goal, Process and Service. Originally, the RGPS proposers suggested that their metamodels should be used together with ontologies [6], [11]. Despite this, it could be interesting for SORE as a research area to check if there are other ways to better describe the RGPS metamodels comparing to the original proposal. This is because ontologies, despite presenting benefits, have also some important drawbacks to this context, such as: (i) difficulty of understanding, maintenance and scalability, hindering ontology model evolution [12] and (ii) ontologies, for instance, when represented using OWL-S, do not execute models in terms of e-services having to be extended or mapped to other methodologies (models and languages, for example) [13].

Therefore, this paper offers some alternatives to the original descriptions (implementations) suggested for the RGPS metamodels by proposing new descriptions that incorporate the benefits of other methodologies established in the literature. Accordingly, this paper proposes the use of the $i^{*}$ Framework [14] to describe Role and Goal layers and the use of Web Services Business Process Execution Language (WS-BPEL) [15] and Web Service Description Language (WSDL) [16] to describe Process and Service layers. Considering these methodologies together, the new approach proposed in this paper is called "Web Services and i* 
Framework"-based RGPS (WS\&i*-RGPS). This paper also presents and discuss a comparison among other SORE approaches in order to evaluate the benefits and drawbacks of the novel WS\&i*-RGPS approach.

This paper is structured as follows: Section II presents the main concepts related to this proposal; Section III outlines WS\&i*-RGPS; Section IV describes works related to this proposal; Section $\mathrm{V}$ presents a comparison among the WS\&i*-RGPS and other approaches in SORE; and Section VI summarizes the conclusions.

\section{BACKGROUND}

This section outlines the methodologies related to the new approach proposed in this paper. Each methodology presented is described in terms of benefits and drawbacks accordingly to the SORE context elicited as the result of a systematic literature review conducted by Souza and Fantinato [17].

\section{A. The RGPS Metamodels}

The RGPS metamodels emerged in the middle of 2007 as an approach to requirements engineering for complex systems distributed in the network, called networked software [6], [10]. The RGPS metamodels propose a way of modeling system requirements by taking account of the SOC paradigm, and defining a modeling architecture that proposes the definition of four layers (Role, Goal, Process and Service). These layers are combined to offer an approach that sets out by analyzing the organizational structure and ends by meeting the specific requirements that are based on e-services [14]. The RGPS proposers suggested that their metamodels should be used together with ontologies [6], [11].

Each layer in the RGPS metamodels can be explained as follows [6], [10], [13], [18]: (i) the Role layer defines the social aspects in the scenario that will be modeled (Roles and Actors are entities defined in RGPS Role layer); (ii) the Goal layer describes stakeholders' requirements as goals and intentions to be achieved, and also defines what the stakeholders expect from the system that is being established; (iii) the Process layer describes the users' requirements according to atomic processes and composed processes executed by the activities; and, (iv) the Service layer describes the execution of the model.

In the SORE context, the main benefits of RGPS are the following: (i) it unifies, in a same approach, both service consumer and service provider perspectives, acting as a bridge between these two highly engaged visions [19]; (ii) it aims at describing custom requirements through different aspects achieved by four hierarchical layers - Role, Goal, and Process Service [6]; and (iii) it is used as a guide for modeling requirements [6], which can be described by different existing methodologies.

The drawbacks of the RGPS, in the SORE context, are presented as follows: $(i)$ it is not supposed to define any methodology for using the metamodels in order to analyze and model requirements; but only defining components and their links in layers [6] and (ii) it was originally designed to be described through ontologies. Therefore, it is a challenge to use other methodologies to describe RGPS layers.
Although the RGPS metamodels have important features in the SORE context, there are still no proposal with alternative solutions or extensions that describe the layers of the solution originally proposed by its authors (i.e. the original proposal with ontologies) [17].

Despite the drawbacks found, the RGPS metamodels were designed for SORE, solving many of the challenges in this research area. Therefore, the RGPS metamodels, if described through methodologies such as $i^{*}$ Framework and WSBPEL/WSDL, could have their benefits leveraged when incorporating the benefits of these methodologies - as proposed in this paper. Originally, RGPS is described with ontologies. Thus, WS\&i*-RGPS proposes an alternative of ontologies descriptions, considering the $i^{*}$ framework and WS-BPEL/WSDL are used in state of practice, increasing the chances of this novel approach to be adopted in the industry.

\section{B. The $i^{*}$ Framework}

The i* Framework was proposed by $\mathrm{Yu}$ [14] as a methodology based on goals and designed to provide a rationale for the environment of organizations and their information systems. It visualizes processes as social players that depend on each other to ensure that their goals are achieved, their tasks performed and their resources used [20]. The framework is composed of two models: (i) the Strategic Dependency Model (SDM), which defines the dependencies between roles and actors and (ii) the Strategic Rationale Model (SRM), which defines the goals and intentions of roles and actors modeled to provide a rationale based on the dependencies modeled with SDM [21].

One of the main assumptions of the $\mathrm{i} *$ Framework is that the entities modeled in an organization should be seen as social actors [22]. The goal of the $i^{*}$ Framework is to provide an understanding of why a system should be developed and not only how to develop it. This fact differentiates it from other approaches in requirements engineering [21]. The systematic literature review presented by Souza and Fantinato [17] demonstrated that only three out of 52 papers found in the SORE context specifically uses the $i^{*}$ Framework in their approaches. However, it was also discovered that, from these total of 52 papers analyzed, $26 \%$ present some solution in SORE that involves a goal-oriented approach - as the $i^{*}$ Framework. This fact confirms the relevance of goal-oriented approaches in the SORE context as used in this proposal.

In the SORE context, the main benefits of the $i^{*}$ Framework are the following: (i) it represents system's requirements whilst keeping information about alternatives [8], [20]; (ii) it assists in managing different aspects of the system specification, such as - stability, support conflict detection, analysis, negotiation, and ability to decompose within the problem domain [4]; (iii) it allows rationalizing on modeled requirements [8]; (iv) it exploits intentional dependencies among actors or strategic roles to assist the description of the system and the organizational environment [14]; (v) it is useful to describe architectural aspects acting as a bridge between requirements and architecture [9]; (vi) it facilitates the composition of components [9]; and (vii) it promotes the abstraction and analysis of a problem domain [9]. 
The drawbacks of the $i^{*}$ Framework, considering SORE context, are the following: $(i)$ it needs to be adapted in order to monitor the system at runtime [8]; (ii) it needs to be combined with other methodologies to offer support to Quality of Service and variability [8]; (iii) it does not promote models' reuse [9]; (iv) it does not promote heterogeneity [9]; $(v)$ it does not present a powerful model configuration support [9]; and (vi) it does not promote the analysis of economic feasibility of the system being modeled [7].

Given such benefits, the $i *$ Framework could contribute in the SORE context. Its drawbacks could be minimized whether they are combined with other methodologies, as proposed in some works in SORE [7], [8], [9].

\section{WS-BPEL/WSDL Languages}

Liegl et al. [23] argued that an approach in SORE should focus on business processes. Vjjayan and Raju [24] stressed that an approach in SORE should include the specifications and analysis of business processes. In this context, it is necessary to adopt an approach that examines the execution of business processes. WS-BPEL and WSDL are languages designed for specifying both executable business processes and the business interaction protocols using web services [25].

WS-BPEL was designed to specify business processes and business interaction protocol using web services (enabled via WSDL descriptions). WS-BPEL provides web service composition so that it can combine services and thus obtain some complex features. Business processes specified by WSBPEL defines the exchange of messages in the web services described by WSDL [15]. In view of this, this paper uses the acronym WS-BPEL/WSDL.

The main benefits of using the WS-BPEL/WSDL languages in the SORE context are the following: (i) they stand out for their maturity and widespread use in industry [26]; (ii) they are considered the standard web service composition form in the industry, strongly recommended by W3C [16]; (iii) they consider implementation aspects of business process by composing e-services [15]; (iv) they describe internal business processes, in organizations, as well as the interaction of internal processes with external processes [27]; (v) they promote requirement verification [27]; and (vi) they promote requirements monitoring and evolution [27], [28].

The drawbacks of using WS-BPEL/WSDL in the SORE context are presented as follows: (i) they do not consider semantic properties, such as pre-conditions and postconditions [6] and (ii) they do not properly address the mapping between high-level requirements descriptions and low-level business processes descriptions [29].

In the systematic literature review presented by Souza and Fantinato [17], from the total of 52 papers analyzed, only two out of them specifically mentioned the use of WSBPEL/WSDL as a possible solution for SORE. On the other hand, around $21 \%$ presented the use of business processes in their approaches, in a general way. Thus, given the benefits mentioned above, the WS-BPEL/WSDL languages could contribute in the SORE context as the execution language of e-services and business processes, despite the presented drawbacks.

\section{RGPS METAMODELS DESCRIBED WITH I* FRAMEWORK AND WS-BPEL/WSDL}

The WS\&i*-RGPS approach proposed in this paper is presented in details in this section. Figure 1 shows an overview of the framework that represents the approach. Notice that the RGPS metamodels are described (i.e., implemented) with the $i^{*}$ Framework and web services technology (through WS-BPEL/WSDL languages). The four RGPS-layers are described as follows:

(i) Role and Goal: these layers are described via the $i^{*}$ Framework. In WS\&i*-RGPS, SDM model from $i^{*}$ Framework is used to describe the Role layer while SRM model, also from i* Framework, is used to describe the Goal layer. The relationship between the Role and Goal layers is established during the SRM specification;

(ii) Process and Service: in WS\&i*-RGPS, these layers are described by WS-BPEL/WSDL. These business processes are executed via web services by means of WSDL descriptions, representing the Service layer. The relationship between the Process and Service layers is directly achieved by the use of WS-BPEL, which orchestrates the composed web services.

In WS\&i*-RGPS, the relationship between the Goal and the Process layers is achieved by mapping rules between the Goal layer components and Process layer components. WS\&i*-RGPS uses the mapping proposed by Séguran, Herbert and Frankova [29]. Decreus, Snoeck and Poels [30] presented a comparison among six approaches that propose the mapping from $i^{*}$ models to business processes. From this comparison, the authors identified the mapping approach proposed by Séguran, Herbert and Frankova [29] as the only one that maps from the $i^{*}$ Framework to the WSBPEL/WSDL languages.

The following subsections provide more information about WS\&i*-RGPS including its four layers and their relationships. The approach is illustrated by means of an example based on the sales of goods in the Brazilian advertising market on the internet.

\section{A. The Role Layer in WS\& $i *-R G P S$}

In WS\&i*-RGPS, the Role layer is described by the SDM model from the $i^{*}$ Framework. Each Role and/or Actor described in the Role layer by the SDM model is specified and refined by the SRM model in the Goal layer. The dependencies are specified and refined in terms of both tasks that have to be executed and resources that have to be consumed. 


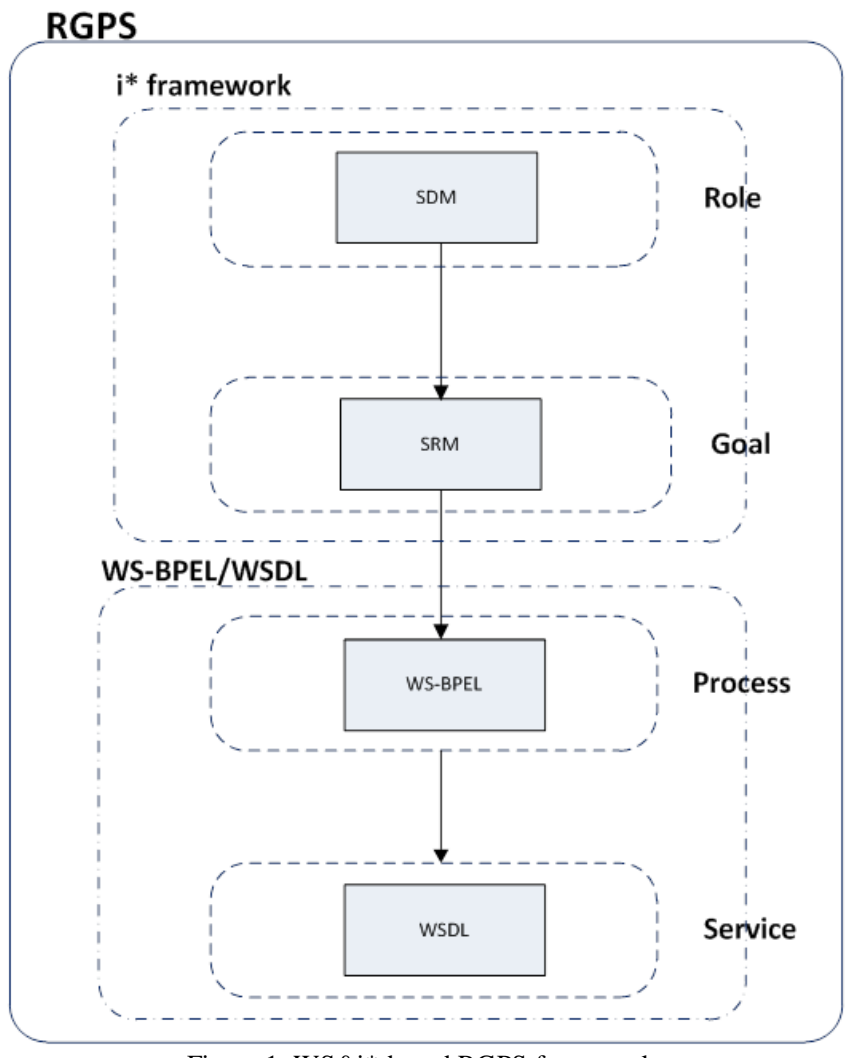

Figure 1. WS\&i*-based RGPS framework.

Figure 2 provides an example of a description of the Role layer based on the $i^{*}$ Framework as proposed in WS\&i*RGPS. This example is based on the Brazilian advertising market for the sale of goods on the internet. The Client role depends on an Agency role to conduct an Advertising Campaign on the internet. On the other hand, the Agency Role depends on a Seller from a Web Portal to be able to hire an advertising service on the internet. Once this service is hired, the Seller Role depends on an Operation Team (OpTeam) to configure and schedule the advertising campaign that has been organized. The OpTeam depends on an Advertiser Server (AdServer) as a resource. This resource is in charge of publishing the advertising campaign on Web Portal.

\section{B. The Goal Layer in WS\& $i^{*}$-RGPS}

In WS\&i*-RGPS, the Goal layer is described by the SRM model, one of the models from the $i^{*}$ Framework. In the RGPS metamodels, alternative tasks are those that can be performed in place of others. And these alternative tasks are also specified in WS\&i*-RGPS with SRM. Moreover, there is a description of softgoals that represent non-functional requirements; the resources and tasks are connected to them via contribution links that indicate whether a task or a resource has had a negative or positive effect in achieving a softgoal [22].

Considering the given Brazilian advertising example, Figure 3 shows the OpTeam role specification in terms of goals and intentions (goals and softgoals) that must be achieved and how to achieve them in terms of resources that can be used and tasks that must be performed. The main goal of the OpTeam is to ensure that the advertising campaign is published on the Web Portal correctly. It is necessary to carry out the task 'Prepare the Publication of an Advertising Campaign' to achieve this. This task is broken down into other three tasks via decomposition links [22] as follows:

(i) Make Digital Medias available: this task consists of making digital media available to the AdServer. It is necessary for the AdServer resource to be available as a software resource to do this. Moreover, the Digital Media must be designed and ready for publication;

(ii) Configure the Advertising Campaign: this task consists of configuring a start date and an end date of publication. It is also necessary to configure the Web Page that will display the advertising campaign and this requires an AdServer to be available;

(iii) Follow up Advertising Campaign Publications: this task consists of ensuring that the advertising campaign is published on the Web Portal as planned by the $\underline{\text { Client. }}$.

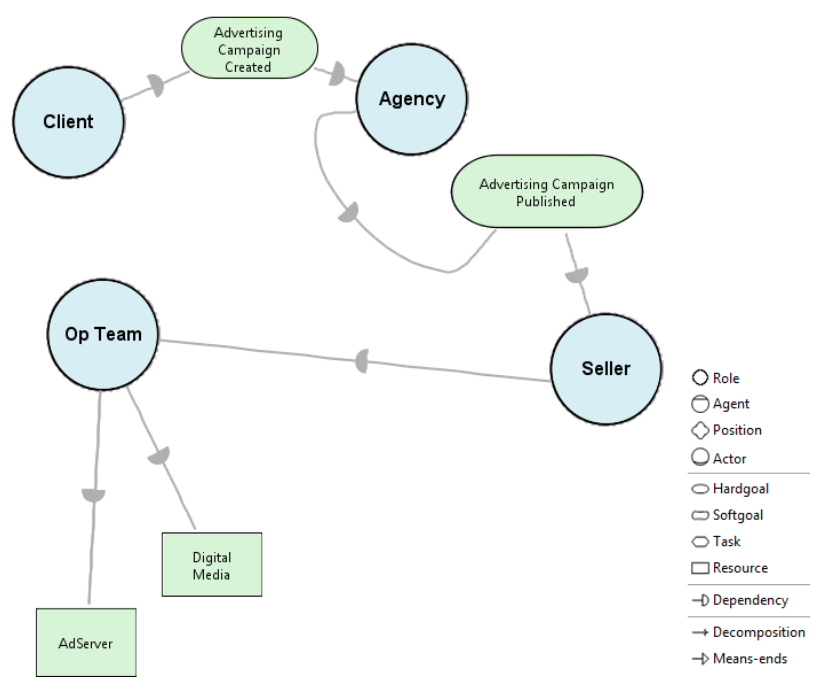

Figure 2. Example of an SDM in WS\&i*-RGPS: description of the Brazilian advertising market for the sale of goods on the internet.

The elements modeled in the Goal layer should be mapped to the Process layer. Some approaches have been already proposed to address the mapping from the SRM model, in $i^{*}$ Framework, to the WS-BPEL language [29], [31]. The mapping rules proposed by Séguran, Herbert and Frankova [29] is adopted in WS\&i*-RGPS because of the evaluation presented by Decreus, Snoeck and Poels [30] and it is designed exclusively to mapping from the $i^{*}$ framework to WS-BPEL/WSDL.

\section{The Process Layer in WS\& $i *-R G P S$}

WS\&i*-RGPS proposes the use of the WS-BPEL/WSDL laguages to model and execute business processes mapped in terms of the composition of web services. The execution of business processes is achieved by structured activities that are connected by control flows, both described by WS-BPEL. Those control flows are executed by web services and 
described by WSDL. There is no need of extra mapping rules between the Process and Service layer, seeing that this mapping is achieved automatically when WS-BPEL/WSDL is used.

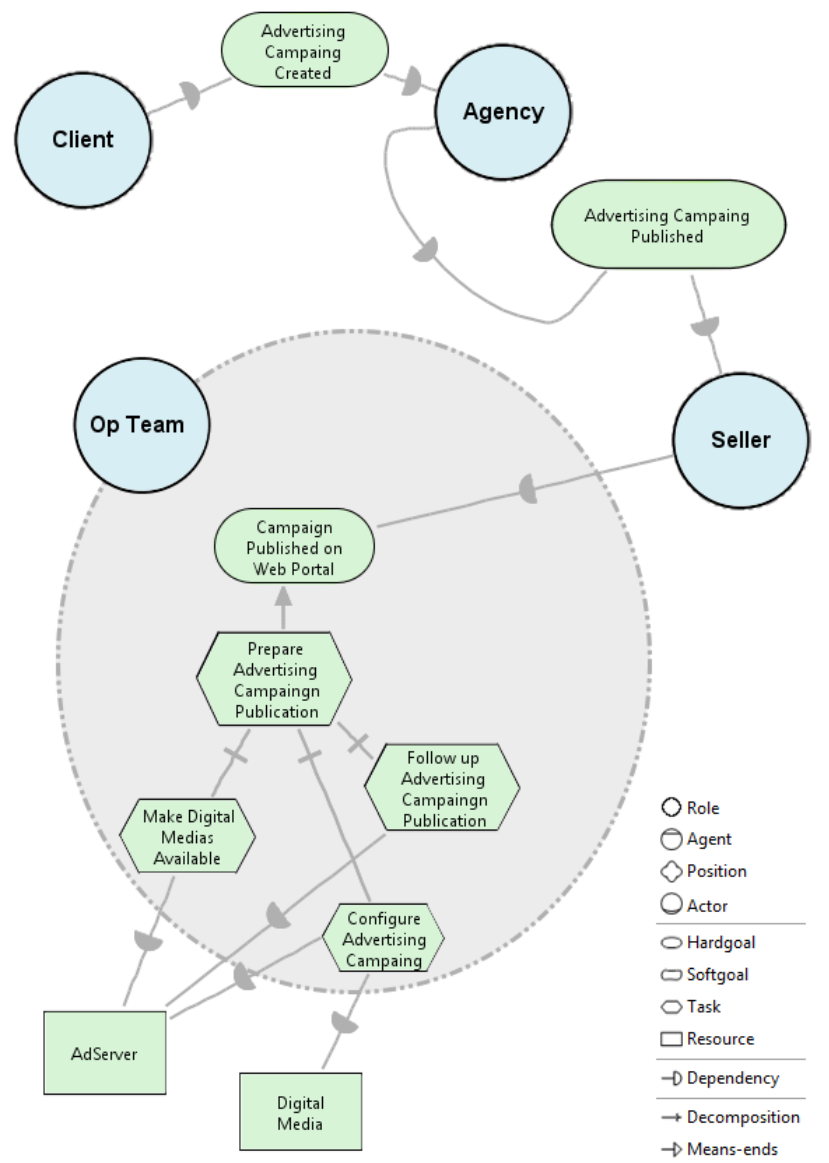

Figure 3. Example of an SRM in WS\&i*-RGPS: Goals for Role Op Team.

The RGPS metamodel components related to the Process layer can be linked to the WS-BPEL/WSDL components. An Atomic Process in the RGPS metamodels, as defined by Wang et al. [6], is described as a Basic Activity in WSBPEL/WSDL. A Composed Process in the RGPS metamodels [6] is described as a Structured Activity - that is a set of Basic Activities executed according to control flows.

These control flows in WS-BPEL/WSDL describe the Control Components in the RGPS metamodels [6]. The Inputs and Outputs in the RGPS metamodels are described by variables in WS-BPEL/WSDL. Nonetheless, there is no component in WS-BPEL/WSDL that describes Preconditions and Post-conditions as suggested in the RGPS metamodels [6].

\section{The Service Layer in WS\& $i *-R G P S$}

The service layer in WS\&i*-RGPS uses web service to describe e-services in a different way from that proposed by the RGPS creators. They proposed the use of semantic web services to describe e-services. However, we strongly believe that using traditional web services to describe Service layer (combined with the use of WS-BPEL) will make it feasible for WS\&i*-RGPS to be adopted by industry as argued by Deng et al. [26].

As in the Process layer, there are components defined by the RGPS Service metamodel that are not supported by the WSDL descriptions. For instance, Pre-conditions and Postconditions are defined in the RGPS Service metamodel but they are not supported by WSDL description. In the case of the service composition that is suggested in the RGPS Service metamodel, this is achieved by a Process layer and not by a Service layer in WS\&i*-RGPS where, the composition of business processes uses a Structured Activity in WSBPEL/WSDL.

\section{RELATED WORK}

Current existing descriptions of the RGPS metamodels use ontologies for requirements specification in all four layers. Therefore, it was found no approach that uses the $i^{*}$ Framework or WS-BPEL/WSDL to describe the RGPS metamodels [17]. It was found an approach extending the RGPS metamodels [13], which uses the guidelines of the requirements engineering methodology Tropos [32] for the specification and requirements analysis in Role and Goals layers. In this approach, the benefits of a goal-oriented methodology are brought to the RGPS metamodels. However, the descriptions of Process and Service layers continued using ontologies.

In the SORE context, in general, Raadt, Gordijn and Yu [7] argued that the use of the $i^{*}$ Framework to explore business strategic goals helps to develop effective systems based on e-services. Recently, Franch et al. [8] used the i* Framework to identify Quality of Service requirements and propose some alternatives to adverse changes by taking account of the SOC paradigm. Pimentel et al. [9] used the i* Framework to ensure the co-evolution between the requirements and architecture by proposing a bridge between requirements modeling and service architecture. This approach established consistency between them.

The SORE approach adopted by Mahbub and Spanoudakis [33] proposes to monitor the requirements at runtime using WS-BPEL/WSDL. Pistore, Roveri and Busseta [34] propose an approach that is oriented to user-requirements to guarantee web service verification.

Shama [35] argues that, considering the recent history of e-business, it is possible to clearly show that the lack of understanding of business models often results in business short-lived and in organizations' failures. Thus, the use of the $i^{*}$ Framework could contribute to a better understanding of the business model resulting in a more assertive requirements engineering. Therefore, the contribution of this paper is to incorporate the benefits of the use of $i^{*}$ and WS-BPEL/WSDL to the RGPS metamodels description seeing that no other work was found combining such methodologies in the SORE context [17]. 


\section{DisCUSSION AND ANALYSIS OF THE WS\&I*- RGPS APPROACH}

In order to evaluate the benefits and the drawbacks of this proposal, WS\&i*-RGPS was compared to other approaches found in literature for the SORE context. The main results of the systematic comparison that was conducted are presented in this section.

The comparison considered the evaluation of three parameters, which were used to identify the boundaries and the scope of the compared approaches:

1) Service consumer and service provider perspectives: approaches in SORE that consider the service consumer perspective address solutions focused on service descriptions from consumers' standpoint; whereas approaches that consider the service provider perspective address solutions focused on service description from providers' standpoint. The ideal scenario suggests that a SORE approach should consider both perspectives [24];

2) Traditional requirements engineering phases: ideal solutions in SORE should cover all traditional requirements engineering phases, such as elicitation, analysis, specification, negotiation, management and verification [36], since this is still a complete task of requirements engineering;

3) Requirements evolution at runtime: ideal solutions in SORE should be able to guarantee requirements evolution at runtime [24] being able to capture any requirements changes and adapt business process and e-services in order to satisfy such changed requirements.

The evaluation presented here compares WS\&i*-RGPS with other SORE approaches found in the systematic literature review conducted by Souza and Fantinato [17]. It is important to highlight that WS\&i*-RGPS was compared only with approaches in SORE that uses the same methodologies, such as the RGPS metamodels, the $i^{*}$ Framework and WSBPEL/WSDL. Table I summarize the approaches selected to this comparative evaluation.

For the RGPS scenario, the approaches that initially proposed the RGPS metamodels have only considered requirements engineering for complex systems distributed in the network - the called Networked Systems [6], [10]. Only in the following works [13], [18], [37], [38], [39] the RGPS metamodels started to be also applied to the specific SORE context. This comparison takes into account only the RGPS approaches applied in the SORE context.

In terms of service consumer and service provider perspectives, Table II shows that most of the approaches address both perspectives. All approaches considered in this comparison consider service consumer perspective. However, four of the approaches in the RGPS metamodels do not address the service provider perspective. WS\&i*-RGPS covers both perspectives.

As presented in Table III, none of the evaluated approaches in SORE covers all phases in traditional requirements engineering. WS\&i*-RGPS does not cover the verification phase. It could be pointed out as an improvement to be done in future work.

TABLE I. APPROACHES IN SORE SELECTED FOR COMPARISON

\begin{tabular}{|c|c|c|}
\hline Methodology & Description & Ref. \\
\hline RGPS & $\begin{array}{l}\text { This approach is a RGPS framework that } \\
\text { addresses the requirements evolution in the } \\
\text { context of e-services. }\end{array}$ & [18] \\
\hline RGPS & $\begin{array}{l}\text { This approach addresses requirements } \\
\text { elicitation considering semantics techniques. }\end{array}$ & [38] \\
\hline RGPS & $\begin{array}{l}\text { This approach addresses a domain-oriented } \\
\text { solution to requirements elicitation and } \\
\text { analysis. Once requirements are analyzed, } \\
\text { this solution allows service discovery based } \\
\text { on requirements. }\end{array}$ & [39] \\
\hline RGPS & $\begin{array}{l}\text { This approach presents a technique to analyze } \\
\text { requirements, and model them in layers. }\end{array}$ & [13] \\
\hline RGPS & $\begin{array}{l}\text { This approach presents a requirements } \\
\text { personalization method. Techniques domain- } \\
\text { oriented are used. }\end{array}$ & [37] \\
\hline$i^{*}$ Framework & $\begin{array}{l}\text { This approach addresses a framework goal- } \\
\text { oriented including a requirement analysis } \\
\text { model, and e-services deployment } \\
\text { guaranteeing evolution and management of } \\
\text { requirements. }\end{array}$ & [8] \\
\hline$i^{*}$ Framework & $\begin{array}{l}\text { This approach presents a solution to model } \\
\text { requirements in order to analyze the } \\
\text { feasibility of business and requirements } \\
\text { alternatives. }\end{array}$ & [7] \\
\hline$i^{*}$ Framework & $\begin{array}{l}\text { This approach addresses the co-evolution } \\
\text { between requirements modeled and } \\
\text { architecture. }\end{array}$ & [9] \\
\hline $\begin{array}{l}\text { WS-BPEL/ } \\
\text { WSDL }\end{array}$ & $\begin{array}{l}\text { This approach presents a verification and } \\
\text { monitoring framework considering web } \\
\text { services. }\end{array}$ & [33] \\
\hline $\begin{array}{l}\text { WS-BPEL/ } \\
\text { WSDL }\end{array}$ & $\begin{array}{l}\text { This approach presents a methodology to } \\
\text { verify requirements implemented in web } \\
\text { services. }\end{array}$ & [34] \\
\hline
\end{tabular}

TABle II. Consumer Perspective X Provider Perspective

\begin{tabular}{|l|l|c|c|}
\hline Ref. & \multicolumn{1}{|c|}{ Methodology } & Consumer & Provider \\
\hline$[18]$ & RGPS & Yes & Yes \\
\hline$[38]$ & RGPS & Yes & No \\
\hline$[39]$ & RGPS & Yes & No \\
\hline$[13]$ & RGPS & Yes & No \\
\hline$[37]$ & RGPS & Yes & No \\
\hline$[8]$ & i* Framework & Yes & Yes \\
\hline$[7]$ & i* Framework & Yes & Yes \\
\hline$[9]$ & i* Framework & Yes & Yes \\
\hline$[33]$ & WS-BPEL/WSDL & Yes & Yes \\
\hline$[34]$ & WS-BPEL/WSDL & Yes & Yes \\
\hline N/A & WS\&i*-RGPS & Yes & Yes \\
\hline
\end{tabular}

Requirements evolution is a challenge in SORE owing to the dynamism addressed in the new paradigm SOC. Therefore, requirements evolution at runtime needs to be considered in SORE approach as argued by Tsai et al. [28]. 
WS\&i*-RGPS considers requirements evolution from requirements model to their implementation with business process and web services. Table IV presents a comparison considering the parameter 3 . The approaches that consider only the early phases of the requirements engineering do not consider requirements evolution at runtime.

TABLE III. TRADITIONAL REQUIREMENTS ENGINEERING PHASES

\begin{tabular}{|c|c|c|c|c|c|c|c|}
\hline Ref. & Methodology & 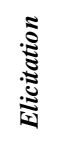 & 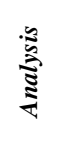 & 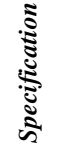 & 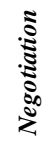 & 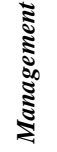 & 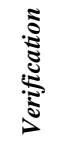 \\
\hline [18] & RGPS & No & No & No & No & Yes & Yes \\
\hline [38] & RGPS & Yes & Yes & No & No & Yes & No \\
\hline [39] & RGPS & Yes & Yes & No & No & No & No \\
\hline [13] & RGPS & No & Yes & Yes & No & No & No \\
\hline [37] & RGPS & No & Yes & No & No & No & No \\
\hline$[8]$ & $i^{*}$ Framework & Yes & Yes & Yes & Yes & Yes & Yes \\
\hline [7] & $i^{*}$ Framework & No & Yes & No & Yes & Yes & No \\
\hline [9] & $i^{*}$ Framework & No & Yes & No & No & Yes & No \\
\hline [33] & WS-BPEL/WSDL & No & No & Yes & No & Yes & Yes \\
\hline [34] & WS-BPEL/WSDL & No & No & Yes & No & No & Yes \\
\hline N/A & WS\&i*-RGPS & Yes & Yes & Yes & Yes & Yes & No \\
\hline
\end{tabular}

TABLE IV. REQUIREMENTS EVOLUTION AT RUNTIME

\begin{tabular}{|l|l|c|c|c|l|c|}
\hline Ref. & Methodology & Evolution & & Ref. & \multicolumn{1}{|c|}{ Methodology } & Evolution \\
\hline$[18]$ & RGPS & Yes & & {$[8]$} & i* Framework & Yes \\
\hline$[38]$ & RGPS & No & & {$[7]$} & i* Framework & Yes \\
\hline$[39]$ & RGPS & No & & {$[9]$} & i* Framework & Yes \\
\hline$[13]$ & RGPS & Yes & & {$[33]$} & WS-BPEL/WSDL & Yes \\
\hline$[37]$ & RGPS & No & & {$[34]$} & WS-BPEL/WSDL & Yes \\
\hline N/A & WS\&i*-RGPS & Yes & & & & \\
\hline
\end{tabular}

As presented in Tables II, III and IV, only the SORE approach presented by Franch et al. [8] fully covers all of comparison parameters evaluated in this paper. WS\&i*RGPS fully covers parameters 1 and 3 and partially covers parameter 2. Regarding parameter 2, the proposed approach does not cover only the verification phase. Concerning RGPS proposes, none of them covers all phases described in parameter 2. Therefore, WS\&i*-RGPS seems to be better than RGPS original approaches because it covers most of phases in requirements engineering (except verification phase). Despite this indicative assessment, further evaluations are required to perform comparisons among the novel approach WS\&i*RGPS and original RGPS approaches.

\section{CONCLUSION}

SORE offers means of overcoming the problems mentioned in the research literature by considering the requirements specifications and analyzing the SOC paradigm. In the SORE context, some approaches are mentioned in the literature that can address these challenges. One of those approaches are the RGPS metamodels, which offer a cooperative and hierarchical approach to model systems requirements based on services by taking account of two standpoints: service consumer and service provider.

WS\&i*-RGPS is a proposed approach in SORE that provides some alternatives to the descriptions of the RGPS metamodels as originally proposed by their creators. This means that WS\&i*-RGPS suggests the use of the i* Framework to describe the Role and Goal layers. Moreover, WS\&i*-RGPS suggests the use of the WS-BPEL/WSDL languages for the Process and Service layers. By doing that, WS\&i*-RGPS attempts to incorporate the benefits of other methodologies already explored in the literature in the RGPS metamodels.

The aim of this paper was to show the benefits of adding the use of the $i^{*}$ Framework to the RGPS metamodels in terms of systems based on services specification, analysis and strategic alignment between the organizations and systems that need to be developed. With regard to the use of the WSBPEL/WSDL, we seek to incorporate the benefits of the WSBPEL/WSDL in the state of practice, and thus increase the chances of WS\&i*-RGPS being adopted by the industry.

This paper also presented a comparison among WS\&i*RGPS and other approaches in SORE. The results showed that WS\&i*-RGPS covers most of the parameter 2. About parameters 1 and 3, WS\&i*-RGPS covers all of them. Comparing to other approaches, the approach presented by Franch et al. [8] covers all parameters including all of traditional requirements engineering phases. However, we deeply believe if the WS\&i*-RGPS is adapted to consider also verification phase, it will be a good option in SORE. It is because of its models and languages combination that potentiated its adoption in the industry. As future work, WS\&i*-RGPS will be adapted to cover all of comparison parameters becoming a completed approach in SORE. Moreover, a comparison among WS\&i*-RGPS and other SORE approaches will be done by using a complex example from the industry.

\section{ACKNOWLEDGMENTS}

This work was supported by The State of São Paulo Research Foundation (Fapesp), Brazil.

\section{REFERENCES}

[1] K. C., Laudon, J. P. Laudon, "Management Information Systems", Pearson Education, 9th Edition, 2011.

[2] M. P. Papazoglou, P. Traverso, S. Dustdar, F. Leymann: "Serviceoriented Computing: A Research Roadmap", International Journal of Cooperative Information Systems, vol. 17, no. 2, pages: 223-255, 2008.

[3] A. van Lamsweerde, "Requirements Engineering in the Year 00: A Research Perspective", In Proceedings of the 22nd International Conference on Software Engineering, 2000, pp. 5-19. 
[4] K. Kontogiannis, G. A. Lewis, D. B. Smith, M. Litoiu, H. Muller, S. Schuster, E. Stroulia, "The landscape of service-oriented systems: a research perspective", In Proceedings of the International Workshop on System Development in SOA Environments, 2007.

[5] Q. Gu, P. Lago, "Exploring service-oriented system engineering challenges: a systematic literature review", Service Oriented Computing and Application, vol. 3, pp. 171-188, 2009.

[6] J. Wang, K. He, B. Li, W. Liu, Peng, W.: "Meta-models of Domain Modeling Framework for Networked Software", In Proceedings of the 6th International Conference on Grid and Cooperative Computing, 2007, p. $878-886$.

[7] B. Raadt, J. Gordijn, E. Yu, "Exploring Web Services from a Business Value Perspective", In Proceedings of the International Conference on Requirements Engineering, 2005, pp. 53-62.

[8] X. Franch, P. Gruünbacher, M. Oriol, B. Burgstaller, D. Dhungana, L. Lopes, J. Marco, J. Pimentel, "Goal-driven Adaptation of ServiceBased Systems from Runtime Monitoring Data", In Proceedings of the International Computer Software and Applications Conference Workshops, 2011, pp. 458-463.

[9] J. Pimentel, J. Castro, E. Santos, A. Finkelstein, "Towards Requirements and Architecture Co-evolution", In Proceedings of the Advanced Information Systems Engineering Workshops, 2012, pp. $159-170$

[10] J, Wang, K. He, P. Gong, C. Wang, R. Peng, B. Li, "RGPS: A Unified Requirements Meta-modeling Frame for Networked Software", In Proceedings of the 3rd International Workshop on Application and Advances of problem frames, 2008.

[11] K. He, P. Liang, R. Peng, B. Li, J. Liu, "Requirement emergence computation of networked software", Frontiers of Computer Science in China, vol. 1: 3, pp. 322-328, 2007.

[12] D. E. O'Leary, "Impediments in the use of Explicit Ontologies for KBS Development", In Proceedings of the International J. HumanComputer Studies, vol. 46, 1997.

[13] B. Hu, K. He, P. Liang, R. Li, "REMO: A RGPS-based Requirements Modeling Process for Service Oriented Architecture", In Proceedings of the International Conference on Networked Computing and Advanced Information Management, 2010, pp. 97 - 102.

[14] E, Yu, "Modeling Strategic Relationships for Process Reengineering", $\mathrm{PhD}$ thesis, Department of Computer Science University of Toronto, 1994.

[15] OASIS Web Services Business Process Execution Language (WSBPEL) TC, http://www.oasis-open.org/

[16] W3C - Web Service Description Language (WSDL), http://www.w3c.com/TR/wsdl

[17] K. S. Souza, M. Fantinato, "Explorando a Engenharia de Requisitos Orientada a Serviços: Uma Revisão Sistemática da Literatura”, In Proceedings of the Simpósio Brasileiro de Sistemas de Informação, 2013. (in Portuguese).

[18] S. Zhang, J. Yin, R. Liu, “A RGPS-Based Framework for ServiceOriented Requirement Evolution of Networked Software", In Proceedings of the International Communication Software and Networks Conference, 2011, pp: 321-325.

[19] F. He, K. He, P. Liang, J. Wang, "On-demand Service-Oriented Architecture and Standardization", In Proceedings of the 8th IEEE International Conference on Service Computing, 2011, pp. 739 - 740.

[20] E. Yu, J. Mylopoulos, “AI Models for Business Process Reengineering”. IEEE Experts - Enterprise Modeling, vol. 11, pp. 1633, 1996.

[21] E. Yu, J. Mylopoulos, N. Maiden, P. Giorgini, "Modeling strategic relationships for process reengineering”. In (eds.). Chapter 2. Social Modeling for Requirements Engineering. MIT Press, 2011.

[22] E, Yu, "Modeling Strategic Relationships for Process Reenginering", $\mathrm{PhD}$ thesis, Department of Computer Science University of Toronto, 1994.

[23] P. Liegl, R. Schuste, M. Zapletal, C. Huemer, H. Werthner, M. Aigner, M. Bernaue, B. Klinger, M. Mayr, R. Mizani, M. Windisch, "[vem:xi:xxx] - A methodology for process based requirements engineering", In Proceedings of the International Conference on Requirements Engineering, 2009, pp. 193-202.

[24] J. Vjjayan, G. Raju, "Service-oriented Requirements Engineering - A new dimension", In Proceedings of the First International Computational Intelligence and Information Technology, pp. 698-704. 2011.

[25] M. Fantinato, M. B. F. Toledo, I. M. S. Gimenes, "WS-Contract Establishment with QoS: An Approach Based on Feature Modeling," International Journal of Cooperative Information Systems, vol. 17, no. 3, pp. 373-407, 2008.

[26] C. Deng, H. Yang, H. Liao, M. Sun , Z. Qiu, “Analysis of WS-BPEL Processes in PRISM". In Proceedings of Fifth International Symposium on Theoretical Aspects of Software Engineering (TASE), 2011, pp.: $199-202$.

[27] M. Pistore1, M. Roveri, P. Busetta, "Requirements-driven verification of Web Services", Electronic Notes in Theoretical Computer Science, pp: 95-108, 2004.

[28] W. T. Tsai, Z. Jin, P. Wang, B. Wu, "Requirement Engineering in Service-Oriented System Engineering", In Proc. of Int. Conf. on eBusiness Engineering,pages: 661 - 668, 2007.

[29] M. Séguran, C. Herbert, G. Frankova, "Secure Workflow Development from Early Requirements Analysis", In Proceedings of the 6th IEEE European Conference on Web Services, 2008.

[30] K. Decreus, M. Snoeck, G. Poels, "Practical Challenges for Methods Transforming i* Goal Models into Business Process Models", In: 17th IEEE International Requirements Engineering Conference, pp. 15-23, 2009.

[31] L. Cysneiros, E. Yu, "Addressing Agent Autonomy in Business Process Management - with Case Studies on the Patient Discharge Process", In Proceeding of Information Resources Management Association Conference, 2004.

[32] E. Yu. Towards modeling and reasoning support for early requirements engineering, In Proccedings RE'97. IEEE Computer Society, 1997.

[33] K. Mahbub, G. Spanoudakis, "A Framework for Requirements Monitoring of Service Based Systems", In Proceedings of the International Conference on Service-Oriented Computing, pp: 84-93, 2007.

[34] M. Pistore, M. Roveri, P. Busseta, "Requirements-Driven Veri cation of Web Services", Journal of Electronic Notes in Theoretical Computer Science, vol. 105, pp. $95-108,2004$.

[35] A. Shama., "Dot-Coms' Coma," J. Systems and Software, vol. 56, no. 1, pp. 101-104, 2001.

[36] I. Sommerville, "Software Engineering, Addison Wesley", 9th Edition, 2010.

[37] H. Chen, K. He, "A Method for Service-Oriented Personalized Requirements Analysis", Journal of Software Engineering and Applications, vol. 4 n. 1, pp. 59-68, 2011.

[38] B. Wen, P. Liang, K. He, "Stakeholders-driven Requirements Semantics Acquisition for Networked Software Systems", In Proceedings of the International Computer Software and Applications Conference, pages: 255-258, 2010.

[39] B. Hu, K. He, H Chen, J. Wang, "Research on the Application of RGPS Domain-based Requirements Elicitation and Analysis for Web Service Discovery", In Proceedings of the International Conference on Semantics, Knowledge and Grid, pp: 290-293, 2009. 